\title{
Disclosure of HIV status: experiences of patients enrolled in an integrated TB and HAART pilot programme in South Africa
}

\author{
Hirut T Gebrekristos ${ }^{1,2 *}$, Mark N Lurie ${ }^{2}$, Nkosinathi Mthethwa ${ }^{3}$ and Quarraisha Abdool Karim ${ }^{1,4}$ \\ ${ }^{1}$ Centre for AIDS Programme of Research in South Africa (CAPRISA), Nelson R Mandela School of Medicine, University of \\ KwaZulu-Natal, Private Bag 7, Congella, Durban 4013, South Africa \\ ${ }^{2}$ Brown University Medical School, Department of Community Health and Medicine and the Miriam Hospital, Box GS-121, \\ 121 S. Main Street, Providence, Rhode Island 02916, United States \\ ${ }^{3}$ Centre for HIVIAIDS Networking (HIVAN), Nelson Mandela School of Medicine, University of KwaZulu-Natal, Umbilo Road, \\ Durban 4041, South Africa \\ ${ }^{4}$ Mailman School of Public Health, Columbia University, 720 W. 168th Street, New York, New York 10032, United States \\ *Corresponding author, e-mail: hgebrekr@jhsph.edu
}

\begin{abstract}
The convergence between the tuberculosis (TB) and HIV epidemics has led to studies investigating strategies for integrated HIV and TB care. We present the experiences of a cohort of 17 patients enrolled in the first integrated TB and HIV treatment pilot programme, conducted in Durban, South Africa, as a precursor to a pivotal trial to answer the question of when to start antiretroviral treatment (ART) in patients co-infected with HIV and TB. Patients' experiences with integrated TB and HIV care can provide insight about the problems or benefits of introducing HIV treatment into existing TB care in resource-constrained settings, where stigma and discrimination are often pervasive and determining factors influencing treatment uptake and coverage. Individual interviews, focus group discussions, and observations were used to understand patients' experiences with integrated TB and HIV treatment. The patients described incorporating highly active antiretroviral therapy (HAART) into their daily routine as 'easy'; however, the patients experienced difficulties with disclosing their HIV status. Non-disclosure to sexual partners may jeopardise safer-sex practices and enhance HIV transmission. Being on TB treatment created a safe space for all patients to conceal their HIV status from those to whom they did not wish to disclose. The data suggest that the context of directly observed therapy (DOT) for TB may have the added benefit of creating a safe space for introducing ART to patients who would benefit most from treatment initiation but who are not ready or prepared to disclose their HIV status to others.
\end{abstract}

Keywords: antiretroviral therapy, co-infection, directly observed therapy, qualitative research, resource-poor settings, sexual behaviour, stigma, treatment issues, tuberculosis

\section{Introduction}

Global solidarity and investment in providing antiretroviral treatment (ART) to those with AIDS in the most affected regions of the world has grown in an unprecedented manner since 2001. Against a backdrop of substantial, real and perceived HIV stigma and discrimination and weakening healthcare systems, the introduction of ART in these settings has not been without challenges. The substantial number of scholarly contributions in the past five years (e.g. Coetzee, Hilderbrand, Boulle, Maartens, Louis, Labatala et al., 2004; Koenig, Leandre \& Farmer, 2004; Ferradini, Jeannini, Pinoges, Izopet, Odhiambo, Mankhambo et al., 2006) reflects the experiences and nature of challenges facing the laudable goal of expanding access to ART to those most in need as espoused by the Global Fund for TB, AIDS and Malaria and the US President's Emergency Plan for AIDS Relief (PEPFAR). Significantly, many of these contributions reflect sustained therapeutic success that is comparable to, and sometimes exceeding, levels achieved in industrialised countries (Coetzee et al., 2004; Koenig et al., 2004; Ferradini et al., 2006; Harries, Schouten \& Libamba, 2006). Notwithstanding these advances and gains, the mortality trajectories in most affected countries remain unaltered as treatment coverage on average remains below 30\% (World Health Organization [WHO], UNAIDS \& UNICEF, 2007).

The convergence of the tuberculosis (TB) and HIV epidemics in resource-constrained settings is reflected in the growing burden of TB cases, including more recent reports of extremely- or multi-drug-resistant cases of TB (Gandhi, Moll, Sturm, Pawinski, Govender, Lalloo et al., 2006; Andrews, Shah, Gandhi, Moll, Friedland \& Tugela Ferry Care and Research Collaboration, 2007; Singh, Upshur \& Padayatchi, 2007). There has been growing attention to integrating ARV and TB treatment services (Harries, Nyangulu, Hargreaves, Kaluwa \& Salaniponi, 2001; Abdool Karim, Abdool Karim, Friedland, Lalloo \& El 
Sadr, 2004; Friedland, Abdool Karim, Abdool Karim, Lalloo, Jack, Gandhi \& El Sadr, 2004; Harries et al., 2006; Blanc, Havlir, Onyebujoh, Thim, Goldfeld \& Delfraissy, 2007); the reasons include better patient outcomes in those co-infected with HIV and TB and improved efficiency (De Cock, Soro \& Coulibaly, 1992; Harries, Kamenya, Subramanyam, Maher, Squire, Wirima et al., 1997; Reid, Scano, Getahun, Williams, Dye, Nunn et al., 2006). Integrating HIV treatment into TB care centres can facilitate the identification of patients eligible for highly active antiretroviral therapy (HAART) and those who stand to benefit most from HAART, with an added benefit of strengthening TB services and management (Corbett, Marston, Churchyard \& De Cock, 2006).

A key challenge in integrating HIV and TB treatment services is establishing the timing of initiating HAART in relation to TB treatment given the antagonistic drug-drug interactions. In preparation for the conduct of a pivotal trial to answer this question, a pilot study of ART provision to a small cohort of HIV-TB co-infected patients was undertaken by Jack, Lalloo, Abdool Karim, Abdool Karim, El Sadr, Cassol \& Friedland (2004). The pilot study enrolled 20 HIV-treatment-naïve patients in Durban, South Africa. Of the 20 patients, 17 successfully completed combined therapy for TB and HIV infections. TB was cured in 17 of the 19 patients with drug-sensitive TB. Among patients completing concurrent TB and HIV treatment, $88 \%$ (15 of 17) had a viral load of $<50$ copies $/ \mathrm{mL}$ and a mean increase of 148 cells $/ \mathrm{mm}^{3}$ in their CD4 cell count (Jack et al., 2004). The authors reported that integrated HIV and TB treatment was well tolerated, safe, and an effective means of delivering HAART.

As part of ongoing evaluations of integrated AIDS and TB treatment services, we utilised the opportunity to interview the cohort of 17 patients from the pilot study by Jack et al. (2004). The purpose of this study was to understand the patient's perspective concerning the obstacles and strengths of introducing HAART in patients co-infected with TB.

\section{Methods}

This study was nested within a pilot study on integrating TB and HIV treatment (i.e. Jack et al., 2004). The eligibility criteria used in selecting patients for the pilot programme was described in detail by Jack et al. (2004); 20 patients were ultimately enrolled. Treatment for TB and HIV was provided concurrently under daily directly observed therapy (DOT). On average, HIV treatment for most patients was initiated four weeks after the initiation of TB treatment. Patients received rifampicin, isoniazid, pyrazinamide and ethambutol, five days a week, by direct observation for the first two months of treatment, together with an ARV drug regimen (didanosine, lamivudine and efavirenz), five days per week, by direct observation and self-administration on weekends. The continuation phase of TB treatment included rifampicin and isoniazid along with HAART. The Cyril Zulu Communicable Diseases Centre in Durban, South Africa, supervised the once-daily dose of TB treatment and HAART during weekdays. After completing TB treatment, the patients self-administered HAART and once a month attended a local HIV clinic at Durban's King Edward VIII Hospital for monitoring (Jack et al., 2004).
From the 20 patients enrolled in the integrated HIV and TB treatment pilot programme, one patient withdrew and two patients were lost to follow-up at different points in the course of their treatment (Jack et al., 2004) and thus before the start of this qualitative study. Of the 17 patients, 13 were females between the ages of 18 and 53 , and the other four were males between the ages of 26 and 39 (Jack et al., 2004). All 17 patients consented to participate in this study.

In-depth qualitative interviews and focus group discussions were conducted in either Zulu or English by investigators trained in qualitative research (N.M. and H.G.). All patients were interviewed, in 2003, at least once, and seven were interviewed at least twice. Two focus group discussions were conducted (the first with five patients and the second involving two participants). The semi-structured interview schedule explored the following items: 1) the patient's decision to have an HIV test; 2) the impact and implications of learning their HIV serostatus; 3 ) their experiences in taking HIV and TB medication; and 4) their coping strategies for dealing with a positive HIV-test result.

The interviews were conducted at different points during treatment and depended on patient availability. We interviewed five patients at less than six months after enrolment, before they completed TB treatment and while they were receiving concurrent HIV treatment. The other 12 patients were interviewed after completing TB treatment, but at different points during their continued HIV treatment (ranging from 3-8 months after the completion of six months of TB treatment). A combination of detailed observations and informal conversations with the participants complimented the tape-recorded interviews with individuals and the focus group discussions. The tape-recorded interviews, conducted in English, were transcribed for analysis. Content analysis of the transcribed interviews was undertaken and organised thematically with respect to the patients' experiences relating to HIV testing, coping with an HIV diagnosis, HAART adherence, status disclosure, social support, and sexual behaviour.

The study was approved by the Ethics Committees of Nelson R Mandela School of Medicine at the University of KwaZulu-Natal (South Africa) and Brown University School of Medicine/the Miriam Hospital (USA).

\section{Results}

\section{Resisting AIDS stigma and negotiating disclosure of HIV status}

Integrated TB and HIV treatment was described as 'easy.' The 'ease' was in part related to the introduction of HAART with the less stigmatised TB treatment. The narratives that patients shared with others about their clinic visits and health revolved around TB. A 39-year-old male said: 'What they know is that I' $m$ taking TB medication...TB is a disease that each and everyone is familiar with so they are not looking too far about having it.' In this implicit comparison with HIV, TB is perceived to receive less 'looking' than HIV. Being treated for TB and attending a TB clinic provided patients with a narrative to resist potential AIDS stigma. This allowed patients to negotiate which persons would come to know their HIV status. For instance, people who the patient 
believed would respond negatively could be told 'I have TB,' while others could be told the truth (that the patient had TB and HIV). Patients also drew a distinction between TB and 'continuous TB' - where having 'continuous TB' that does not respond to treatment was understood to be 'AIDS.' When patients on integrated TB and HIV treatment started to recover physically, people in the community did not link their TB infection to AIDS. Thus, recovering from TB worked to reinforce images that are inconsistent with AIDS illness and helped to remove people's inquiry or 'looking.'

Like 'continuous TB,' the image of weight loss is linked with AIDS. Gaining weight also worked to transform people's 'looking' to embrace a patient's improved health. Before participating in integrated TB and HIV treatment most patients described being 'very sick and thin.' Patients gained an average of $20 \mathrm{~kg}$ over the course of concurrent treatment (after six months). Many acknowledged this with great enthusiasm: 'I'm getting fat!' The participants' weight gain received significant attention from people around them; one patient said, 'I used to isolate myself at home because too many people were asking about why I was getting so thin.' She then described that weight loss leads to 'fingerpointing' (assumptions of AIDS illness) and she was 'scared of people's reactions.' She reported that being on treatment had helped her regain her weight, and now, 'People are asking me how I am gaining [weight].' Another patient (35-year-old female) described that people thought her weight loss was 'AIDS, but [now that I am gaining weight] they are saying you are looking good...it's not AIDS!' Thus these narratives relating fears of AIDS stigma illustrate the importance of gaining weight, as gaining weight confirmed for others that it was 'not AIDS' and liberated patients from being labelled 'AIDS.'

Liberation from being labelled 'AIDS' and their participation in HAART exposed patients to different ways of interacting with people around them. Patients found themselves counselling people or taking free condoms and pamphlets for others from the TB clinic. One patient said, 'I saw the pamphlets can be more helpful for the community... before I did nothing because I was not connected with the situation...I had not been counselled.' When people spoke negatively about being HIV-positive, another patient said, 'I just counsel them and make it clear on the mind.' These personal initiatives highlight how resisting individual stigma may liberate patients into quietly advocating against societal AIDS stigma.

\section{The challenges of AIDS stigma and non-disclosure}

Using their concurrent TB treatment, all HIV patients were able to resist AIDS stigma and to negotiate disclosure of their status. However, 'hiding' their HIV status with TB did not remove other challenges. Fears of AIDS stigma were at the centre of the challenges they faced with disclosing their HIV status. The patients' decisions to disclose their HIV status centred on two broad issues: 1) their expectations of support from family and friends, and 2) personal preparedness. Those who had disclosed their HIV serostatus felt that doing so provided support: '[l told him] when you have problems he helps. I told his mother because she has a good heart.' Fears of being labelled 'AIDS' restricted patients in the ways they talked about HIV. Participants revealed that HIV is different because 'you can't trust anybody' with HIV. Participants suggested that non-disclosure is 'better' because 'people will not understand.' For example, one patient's husband '...says that people who are HIV should die and the government should not help them.' A patient who had not disclosed to anyone said, 'People [would] point at me. I don't want to be a bad example.' Other patients did not disclose to their family because they did not want to 'worry them.' This was particularly the case in families that had already experienced AIDS-related deaths in the recent past.

Even with supportive environments, some patients struggled with the personal discomforts of disclosure, ranging from feelings of shame to guilt for being 'AIDS.' For example, 'I haven't told anyone...no, they [family] would be supportive [but] it's difficult to talk about HIV.' Being an HIV-positive person restricted how patients choose to talk about HIV or AIDS among friends and family. One patient (28-year-old male) said, 'If someone talks about HIV to me...I would feel guilty because I know I am HIV-positive...I [would think] maybe this person sees that I am HIV-positive, that's why I don't want to talk about HIV.'

Patients who had not disclosed their HIV status had to conceal HAART from those around them. In the interviews, we explored whether or not hiding medication was related to treatment default. Patients living on their own reported concealing their medicines and their HIV serostatus from family and friends without difficulties. One patient reported, 'I don't want many questions, that's why I [like] living alone.' Most people, however, shared their living space with others and they told their family that the medications were 'all' for TB. Only two of the patients shared their living space with their partners, and one of them had not disclosed to her partner. Even if the participants were sharing their living space with people who were not aware of their HIV serostatus and treatment, in the context of TB treatment, the proximity of family or friends who were 'making sure' they were taking their TB treatment was reported to simultaneously serve as a reminder for HAART. After TB treatment completion and cure, some patients who had not disclosed continued to tell family and friends that they were on TB treatment, thereby avoiding disclosing their HIV status. The patients found it fairly easy to say, for example, 'Don't touch...they [the HIV tablets] are for TB.' Patients who had other health problems also used that to hide their HIV status and treatment. For example, one patient told her partner who lived with her that she was attending a clinic for evaluation and treatment for arthritis.

We also explored whether hiding one's HIV status from sexual partners resulted in unsafe sex. Only three participants had disclosed to their sexual partners. The participants described frustrations around using condoms consistently. Though participants understood the value of sharing their HIV status with sexual partners, fears of being rejected or fears of experiencing violence remained obstacles for disclosure and negotiating safer-sexual practices:

'It's difficult if you just tell him that...we must use condoms. He thinks you have another boyfriend or 
another affair. There are arguments [about using condoms]...but, I don't tell him that I'm HIV-positive. I told him I want to use a condom to be safe...I don't like it [condoms], but I have to because I'm HIV-positive' (33-year-old female).

'I'm afraid to sleep with her...sometimes she asks me why don't you want to have sex with me and I don't respond to her' (27-year-old male).

'I'm scared to tell him. He has a gun' (38-year-old, female).

Non-disclosure was cited for inconsistent condom use. A 38-year-old woman commented, 'What can I say [when asked 'let's not use condoms'] if I haven't told about [my] HIV [status]'; she also reported how the arguments and disagreements about condom use were frustrating: 'It's too much.'

\section{Discussion}

The physical manifestations of TB and AIDS are common and well known, particularly in constrained settings: the dramatic and sudden weight loss, physical weakness and changes in physical appearance cannot be disguised. While TB is curable and less stigmatised, the narratives around HIV infection are associated with death, hopelessness, and stigma. The similarities and differences in the narratives about experiences with TB or HIV infection support the idea of integrated TB and HIV treatment. Participating in integrated TB and HIV treatment within a TB clinic allowed patients to resist AIDS stigma and negotiate disclosure of their HIV status.

The challenges that AIDS stigma presents for effective HIV prevention and care have been described in the literature (e.g. Parker \& Aggleton, 2003; Campbell, Foulis, Maimane \& Sibiya, 2005; Norman, Chopra \& Kadiyala, 2007). The narratives related in this study suggest that introducing HAART in the TB-treatment setting may offer opportunities for building interventions to reduce HIV-related stigma at the individual and community levels. The difficulties that patients faced with disclosure were not unique to the provision of HAART in the context of a TB clinic. However, the TB-treatment infrastructure may provide opportunities for patients to negotiate which persons will come to know their HIV status. A common theme in the interviews was the use of TB medications to hide HIV status and HIV medications. From the patient's perspective, 'hiding behind TB' was a major strategy for coping with their HIV status and for resisting AIDS stigma. Liechty \& Bangsberg (2003) suggest that daily ART under directly observed therapy (DOT) may lead to the stigmatisation of patients on treatment. The findings of this study suggest that HAART administered under DOT and concurrently with TB-DOT may present an opportunity for patients to cope with their HIV status. Participating in TB-DOT for six months, where patients can engage daily with healthcare providers, may be useful for introducing interventions that support HIV disclosure in ways that advance prevention and care strategies.

We should also note interventions at the level of the individual (to reduce stigma) have limitations (Parker \& Aggleton, 2003). Although using TB treatment to hide one's
HIV status provides short-term individual protection from AIDS stigma, the alleviation of stigma must operate at the social level, where stigma is constructed and reproduced (Parker \& Aggleton, 2003). Initiatives to promote empowerment and community involvement are important for addressing stigma at the societal level (Parker \& Aggleton, 2003; Campbell et al., 2005; Norman et al., 2007). In South Africa, one of the ways that empowerment and community involvement have been promoted is through the activities of the Treatment Action Campaign (TAC) (Campbell et al., 2005; Norman et al., 2007). The patients interviewed in this study were not involved with TAC or other groups, but the patients described a change in the way they interacted with relatives and friends, where they found themselves taking free condoms or pamphlets on HIV and counselling others about HIV. Though these activities were limited by non-disclosure, organising around these personal initiatives to build collective efforts against AIDS stigma, within and outside TB clinics, may help alleviate AIDS stigma at the individual and societal levels.

Where living space is limited, as is the case with the majority of participants, concealing HIV medication is challenging in instances where HIV-status disclosure has not occurred. Supporting disclosure should be an integral component in the continuum of care for persons with HIV infection. In this study, there was a clear distinction between individual challenges to disclosure and those projected by family and friends. Supporting patients who have personal difficulties with coping with their HIV-seropositive status is one of the objectives of VCT programmes. The frustrations experienced with non-disclosure which centre on patients' inability to cope with their HIV status may create obstacles for HAART implementation and HIV-prevention strategies (De Cock, Bunnell \& Mermin, 2006).

Disclosure may also have implications for treatment adherence. However, in this study there were no significant challenges reported in relation to taking HAART even when people did not disclose. We suggest that a combination of factors, including limited knowledge of HAART in the community and the relatively small number of people participating in HIV treatment, allows patients to effectively conceal their HIV status and HAART from family and friends. Although non-disclosure was not reported to influence patients' commitment to HAART, their abilities to conceal their HIV status and treatment without having to confront the perceived and observed consequences of being HIV-seropositive may be significantly challenged as treatment becomes widely available. Efforts to increase access to HAART must therefore actively encourage disclosure of one's HIV-positive status to family, friends, and partners.

The limited data available on HIV disclosure in Africa suggests that non-disclosure may reduce emotional support to cope with an HIV-positive status and the capacity to introduce safer-sexual behaviours into relationships (Keogh, Allen \& Almedal, 1994; Antelman, Fawzi \& Kaaya, 2001; Grinstead, Gregorich, Choi, Coates \& Voluntary HIV-1 Counselling and Testing Efficacy Study Group, 2001; Maman, Mbwambo, Hogan, Kilonzo \& Sweat, 2001; Luchters, Sarna, Geibel, Chersich, Munyao, Kaai et al., 2008). The experiences described here indicate that 
non-disclosure may jeopardise safe-sexual behaviour. The dynamics of disclosure to sexual partners requires further attention in building a sound continuum of care and prevention for persons infected with HIV.

Limitations with the study design should be noted. The interviews were not predetermined. The timing for the initial and following-up interviews did not occur at the same time for all patients. The participants in this study included all patients who continued to remain in the pilot study and who therefore completed concurrent TB treatment and HAART. Because the narratives of patients who either withdrew or who were lost to follow-up may differ from those who participated in this study, it is unclear how this limitation may have influenced the overall themes. In addition, the interviews were collected in 2003 before HAART roll-out occurred in the public sector in South Africa. Thus, the perspectives presented may differ slightly from current thinking. However, research findings on the topic found in recent publications would suggest the narratives presented remain relevant (e.g. Daftary, Padayatchi \& Padilla, 2007; Norman et al., 2007).

Acknowledgements - CAPRISA is part of the Comprehensive International Program of Research on AIDS (CIPRA) and is supported by the National Institute of Allergy and Infectious Disease (NIAID), the National Institutes of Health (NIH) and the US Department of Health and Human Services (DHHS) (grant no. 1 U19 Al51794). Support was also provided by the Columbia University-Southern Africa Fogarty AIDS International Training and Research Program (grant no. D43 TW00231) and by a Research Supplement for Under-represented Minorities under the Brown/ Tufts/Lifespan Centre for AIDS Research (CFAR) at Brown University, Rhode Island, USA (grant no. P30 Al42853-05S1). Additional support came from a T-32 training grant awarded by the National Institute of Drug Abuse to the Miriam Hospital (grant no. 5 T32 DA113911) and a K-01 from the National Institute of Mental Health awarded to Mark Lurie (grant no. 1 K01 MH069113-01A1). We thank the staff of the pilot programme, which was supported by grants from the Irene Diamond Fund and Doris Duke Charitable Foundation, including Christopher Jack, Colleen Jiyani, Vikesh Naidoo and all the participants. We also thank Gerald Friedland, principal investigator of the pilot study, for providing valuable comments on an earlier draft of this paper.

The authors - Hirut Gebrekristos (MPH) completed a predoctoral research fellowship at the Centre for the AIDS Programme of Research in South Africa (CAPRISA) and is currently working on a doctoral degree in epidemiology at Johns Hopkins University (USA).

Mark Lurie $(\mathrm{PhD})$ is a social epidemiologist who has been conducting research on HIVIAIDS, TB and STIs in South Africa since 1995.

Nkosinathi Mthethwa (BSc) is coastal region territory supervisor with Sandoz, TB and Tender Division (South Africa).

Quarraisha Abdool Karim (PhD) is Director of the Columbia University-Southern Africa Fogarty AIDS International Training and Research Program and the Associate Scientific Director of the Centre for the AIDS Programme of Research in South Africa (CAPRISA).

\section{References}

Abdool Karim, S.S., Abdool Karim, Q., Friedland, G., Lallo, U. \& El Sadr, W. (2004) Implementing antiretroviral therapy in resourceconstrained settings: opportunities and challenges in integrating HIV and tuberculosis care. AIDS 18, pp. 975-979.
Andrews, J.R., Shah, N.S., Gandhi, N., Moll, T., Friedland, G. \& Tugela Ferry Care and Research (TF CARES) Collaboration (2007) Multidrug-resistant and extensively drug-resistant tuberculosis: implications for the HIV epidemic and antiretroviral therapy rollout in South Africa. Journal of Infectious Diseases 196(supplement 3), pp. S482-490.

Antelman, G., Fawzi, M.C.S. \& Kaaya, S. (2001) Predictors of HIV-1 sero-status disclosure: a prospective study among HIV-infected pregnant women in Dar es Salaam, Tanzania. AIDS 15, pp. 1865-1874.

Blanc, F.X., Havlir, D.V., Onyebujoh, P.C., Thim, S., Goldfeld, A.E. \& Delfraissy, J.F.(2007) Treatment strategies for HIV-infected patients with tuberculosis: ongoing and planned clinical trials. Journal of Infectious Diseases 196(supplement), pp. S46-S51.

Campbell, C., Foulis, C.A., Maimane, S. \& Sibiya, Z. (2005) 'I have an evil child at my house': Stigma and HIVIAIDS management in a South African community. American Journal of Public Health 95, pp. 808-815.

Coetzee, D., Hilderbrand, K., Boulle, A., Maartens, G., Louis, F., Labatala, V., Reuter, H., Ntwana, N. \& Goemaere, E. (2004) Outcome after two years of providing antiretroviral treatment in Khayelitsha, South Africa. AIDS 18, pp. 887-895.

Corbett, E.L., Marston, B., Churchyard, G.J. \& De Cock, K.M. (2006) Tuberculosis in sub-Saharan Africa: opportunities, challenges, and change in the era of antiretroviral treatment. The Lancet 367, pp. 926-937.

Daftary, A., Padayatchi, N. \& Padilla, M. (2007) HIV testing and disclosure: a qualitative analysis of TB patients in South Africa. AIDS Care 19, pp. 572-577.

De Cock, K., Bunnell, R. \& Mermin, J. (2006) Unfinished business: expanding HIV testing in developing countries. New England Journal of Medicine 354, pp. 440-442.

De Cock, K., Soro, B. \& Coulibaly, I.M. (1992) Tuberculosis and HIV infection in sub-Saharan Africa. Journal of the American Medical Association 268, pp. 1581-1587.

Ferradini, L., Jeannini, A., Pinoges, L., Izopet, J., Odhiambo, D., Mankhambo, L., Karungi, G., Szumilin, E., Balandine, S., Fedida, G., Carieri, M.P., Spire, B., Ford, N., Tassie, J., Guerin, P.J. \& Brasher, C. (2006) The Lancet 367, pp. 1335-1342.

Friedland, F., Abdool Karim, S., Abdool Karim, Q., Lalloo, U., Jack, C., Gandhi, N. \& El Sadr, W. (2004) Utility of tuberculosis directly observed therapy programs as sites for access to and provision of antiretroviral therapy in resource-limited countries. Clinical Infectious Diseases 38, pp. S421-S428.

Gandhi, N.R., Moll, A., Sturm, A.W., Pawinski, R., Govender, T., Lalloo, U., Zeller, K., Andrews, J. \& Friedland, G. (2006) Extensively drug-resistant tuberculosis as a cause of death in patients co-infected with tuberculosis and HIV in a rural area of South Africa. The Lancet 368, pp. 1575-1580.

Grinstead, O.A., Gregorich, S.E., Choi, K.H., Coates, T. \& Voluntary HIV-1 Counselling and Testing Efficacy Study Group (2001) Positive and negative life events after counselling and testing: the voluntary HIV-1 counselling and testing efficacy study. AIDS 15 , pp. $1045-1052$.

Harries, A.D., Kamenya, A., Subramanyam, V.R., Maher, D., Squire, S.B., Wirima, J.J., Nyangulu, D.S. \& Nunn, P. (1997) Transactions of the Royal Society of Tropical Medicine and Hygiene 91, pp. 416-419.

Harries, A.D., Nyangulu, D.S., Hargreaves, N.J., Kaluwa, O. \& Salaniponi, F.M. (2001) Preventing antiretroviral anarchy in sub-Saharan Africa. The Lancet 358, pp. 410-414.

Harries, A.D., Schouten, E.J. \& Libamba, E. (2006) Scaling up antiretroviral treatment in resource-poor settings. The Lancet 367, pp. 1870-1872.

Jack, C., Lalloo, U., Abdool Karim, Q., Abdool Karim, S.S., El Sadr, W., Cassol, S. \& Friedland, G. (2004) A pilot study of 
once-daily antiretroviral therapy integrated with tuberculosis directly observed therapy in a resource-limited setting. Journal of Acquired Immune Deficiency Syndromes 36, pp. 929-934.

Keogh, P., Allen, S. \& Almedal, C. (1994) The social impact of HIV infection on women in Kigali, Rwanda: a prospective study. Social Science and Medicine 38, pp. 1047-1053.

Koenig, S.P., Leandre, F. \& Farmer, P.E. (2004) Scaling-up HIV treatment programmes in resource-limited settings: the rural Haiti experience. AIDS 18, pp. S21-S25.

Liechty, C.A. \& Bangsberg, D.R. (2003) Doubts about DOT: antiretroviral therapy for resource-poor countries. AIDS 17, pp. 1383-1387.

Luchters, S., Sarna, A., Geibel, S., Chersich, M.F., Munyao, P., Kaai, S., Mandaliya, K.N., Shikely, K.S., Rutenberg, N. \& Temmerman, M. (2008) Safer sexual behaviours after 12 months of antiretroviral treatment in Mombasa, Kenya: a prospective cohort. AIDS Patient Care and STDs 22(7), pp. 587-594.

Maman, S., Mbwambo, J., Hogan, N.M., Kilonzo, P. \& Sweat, M. (2001) Women's barriers to HIV-1 testing and disclosure: challenges for HIV-1 voluntary counselling and testing. $\underline{\text { AIDS }}$ Care 13, pp. 595-603.
Norman, A., Chopra, M. \& Kadiyala, S. (2007) Factors related to HIV disclosure in two South African communities. American Journal of Public Health 97, pp. 1775-1781.

Parker, R. \& Aggleton, P. (2003) HIV and AIDS-related stigma and discrimination: a conceptual framework and implications for action. Social Science and Medicine 57, pp. 13-24.

Perriens, J.H., Mukadi, Y. \& Nunn, P. (1991) Tuberculosis and HIV infection: implications for Africa. AIDS 5(supplement 1), pp. S127-S133.

Reid, A., Scano, F., Getahun, H., Williams, B., Dye, C., Nunn, P., De Cock, K.M., Hankins, C., Miller, B., Castro, K.G. \& Raviglione, M.C. (2006) Towards universal access to HIV prevention, treatment, care, and support: the role to tuberculosis/HIV collaboration. The Lancet Infectious Diseases 6, pp. 483-495.

Singh, J.A., Upshur, R. \& Padayatchi, N. (2007) XDR-TB in South Africa: no time for denial or complacency. PLoS Medicine 4(1). Online at: e50 doi:10.1371/journal.pmed.0040050.

World Health Organization (WHO), UNAIDS \& UNICEF (2007) Towards Universal Access: Scaling up priority HIVIAIDS interventions in the health sector. Progress Report, April 2007. Geneva, WHO. 